\title{
Hot and cold executive functions in youth with psychotic symptoms
}

\author{
L. E. MacKenzie ${ }^{1,2,3}$, V. C. Patterson ${ }^{2,3}$, A. Zwicker ${ }^{2,3,4}$, V. Drobinin ${ }^{2,3,5}$, H. L. Fisher ${ }^{6}$, S. Abidi ${ }^{2,7}$, \\ A. N. Greve ${ }^{8}$, A. Bagnell ${ }^{2,7}$, L. Propper ${ }^{2,7}$, M. Alda ${ }^{2,3}$, B. Pavlova ${ }^{2,3}$ and R. Uher ${ }^{1,2,3,4,5,6,7 *}$ \\ ${ }^{1}$ Department of Psychology and Neuroscience, Dalhousie University, Halifax, Nova Scotia, Canada \\ ${ }^{2}$ Department of Psychiatry, Dalhousie University, Halifax, Nova Scotia, Canada \\ ${ }^{3}$ Nova Scotia Health Authority, Halifax, Nova Scotia, Canada \\ ${ }^{4}$ Department of Pathology, Dalhousie University, Halifax, Nova Scotia, Canada \\ ${ }^{5}$ Department of Medical Neuroscience, Dalhousie University, Halifax, Nova Scotia, Canada \\ ${ }^{6}$ Social, Genetic \& Developmental Psychiatry Centre, Institute of Psychiatry, Psychology \& Neuroscience, King's College London, UK \\ ${ }^{7}$ IWK Health Centre, Halifax, Nova Scotia, Canada \\ ${ }^{8}$ Psychosis Research Unit, Aarhus University Hospital, Risskov, Denmark
}

Background. Psychotic symptoms are common in children and adolescents and may be early manifestations of liability to severe mental illness (SMI), including schizophrenia. SMI and psychotic symptoms are associated with impairment in executive functions. However, previous studies have not differentiated between 'cold' and 'hot' executive functions. We hypothesized that the propensity for psychotic symptoms is specifically associated with impairment in 'hot' executive functions, such as decision-making in the context of uncertain rewards and losses.

\begin{abstract}
Methods. In a cohort of 156 youth (mean age 12.5, range 7-24 years) enriched for familial risk of SMI, we measured cold and hot executive functions with the spatial working memory (SWM) task (total errors) and the Cambridge Gambling Task (decision-making), respectively. We assessed psychotic symptoms using the semi-structured Kiddie Schedule for Affective Disorders and Schizophrenia interview, Structured Interview for Prodromal Syndromes, Funny Feelings, and Schizophrenia Proneness Instrument - Child and Youth version.
\end{abstract}

Results. In total 69 (44.23\%) youth reported psychotic symptoms on one or more assessments. Cold executive functioning, indexed with SWM errors, was not significantly related to psychotic symptoms [odds ratio (OR) 1.36, 95\% confidence interval (CI) 0.85-2.17, $p=0.204)$. Poor hot executive functioning, indexed as decision-making score, was associated with psychotic symptoms after adjustment for age, sex and familial clustering (OR 2.37, 95\% CI 1.25-4.50, $p=0.008$ ). The association between worse hot executive functions and psychotic symptoms remained significant in sensitivity analyses controlling for general cognitive ability and cold executive functions.

Conclusions. Impaired hot executive functions may be an indicator of risk and a target for pre-emptive early interventions in youth.

Received 22 October 2016; Revised 16 April 2017; Accepted 27 April 2017; First published online 7 June 2017

Key words: Cold executive functions, decision-making, hot executive functions, offspring of affected parents, psychotic symptoms, severe mental illness, youth at-risk.

\section{Introduction}

Psychotic symptoms, including hallucinations and delusions, are the hallmark of severe mental illness (SMI), such as schizophrenia and major mood disorders. Psychotic symptoms also commonly occur in individuals without SMI, and are especially common in childhood and adolescence. General population studies have found that $10-17 \%$ of children and $7.5 \%$ of adolescents report unusual experiences that are undistinguishable from delusions and hallucinations

\footnotetext{
* Address for correspondence: R. Uher, 5909 Veterans' Memorial Lane, Halifax, NS, B3H 2E2, Canada.

(Email: uher@dal.ca)
}

that occur in SMI (Kelleher et al. 2012a, b). Psychotic symptoms in childhood are associated with family history of psychotic and mood disorders (Polanczyk et al. 2010; Zammit et al. 2013, 2014) and predict a range of psychiatric disorders in adulthood, including schizophrenia, other forms of SMI, as well as suicide attempts (Poulton et al. 2000; Welham et al. 2009; Fisher et al. 2013). Therefore, psychotic symptoms in childhood and adolescence may represent early manifestations of liability, and understanding their underlying mechanisms may provide clues to the etiology and prevention of SMI.

SMI is associated with impairments in cognitive ability, especially in executive functions (Westerhausen et al. 2011; Bora et al. 2013; Snyder, 2013; Bora \&

This is an Open Access article, distributed under the terms of the Creative Commons Attribution licence (http://creative commons.org/licenses/by/4.0/), which permits unrestricted re-use, distribution, and reproduction in any medium, provided the original work is properly cited. 
Pantelis, 2015). The presence of a milder degree of impairment among unaffected biological relatives of individuals with SMI suggests that cognitive functions are associated with the genetic liability to SMI (Bora et al. 2009; Jameson et al. 2011). To uncover the etiological mechanisms behind the development of SMI, it may be important to determine which types of cognitive functions are most strongly related to psychopathology.

Executive function is a broad term for higher order cognitive functions typically associated with the frontal lobes, such as initiating, planning, working memory, cognitive flexibility, monitoring, decision-making, and the ability to solve novel problems (Chan et al. 2008). Rather than examining executive functions as a single domain, it may be important to distinguish between 'cold' and 'hot' executive functions (Rushworth \& Owen, 1998; Grafman \& Litvan, 1999). 'Cold' executive functions are emotion-independent and logically-based. A typical example of cold executive function is working memory, which describes the ability to hold and manipulate a number of items of information in active memory. 'Hot' executive functions involve emotion, desires, motivation, and rewards. A typical example of hot executive function is decision-making, where an individual is making choices with potentially rewarding or aversive consequences (Bechara et al. 1999, 2000; Roiser \& Sahakian, 2013).

Psychotic symptoms are emotionally salient and distressing to individuals who experience them. Cognitive and neural models of psychosis point to emotional appraisal and salience as the key mechanisms that can lead to the experience of psychotic symptoms (Underwood et al. 2015; Reininghaus et al. 2016). Therefore, we expect that impairments in hot executive functions will be more closely related to the propensity to experience psychotic symptoms. Previous studies in youth with psychotic symptoms have identified cognitive impairment in cold executive functions, including processing speed and working memory (Cullen et al. 2010; Blanchard et al. 2010, Kelleher et al. 2012a, b; Dickson et al. 2014). The relationship between hot executive functions and the propensity for psychotic symptoms has not yet been explored. In the present study, we seek to close this gap in knowledge by simultaneously assessing cold and hot executive functions in young people who do or do not experience psychotic symptoms. We tested the hypothesis that impaired performance on measures of hot executive functioning would be specifically associated with psychotic symptoms.

\section{Methods}

\section{Participants}

We explored the relationship between hot and cold executive functions and psychotic symptoms in 156 participants aged 7-24, recruited as part of the Families Overcoming Risks Building Opportunities for Wellbeing (FORBOW) study, a cohort of individuals enriched for familial high risk of SMI (Uher et al. 2014). Sons and daughters of parents with SMI were recruited through mental health professionals in Nova Scotia, Canada, who inquired whether patients with psychotic and major mood disorders had children in the eligible age range. Offspring participants were included regardless of whether they had current psychopathology or not. Partnership with the Nova Scotia Department of Community Services enabled enrolment and follow-up of all biological offspring, including sons and daughters not in the care of their biological parents. Offspring of healthy control parents were recruited through local school boards. The present sample included $126(81 \%)$ offspring of parents with SMI and $30(19 \%)$ offspring of control parents without SMI (Table 1). Exclusion criteria were brain injury or severe intellectual disability of a degree that would invalidate assessment. The Research Ethics Board of the Nova Scotia Health Authority approved the study protocol. All participants with capacity provided written informed consent. For children who did not have capacity to make an informed decision, a substitute decision-maker (parent or legal guardian) provided written informed consent and the child provided assent.

\section{Parent assessment}

Diagnostic and Statistical Manual (DSM)-IV and DSM-5 diagnoses of mental disorders and family history of psychotic symptoms were established with the Schedule for Affective Disorders and Schizophrenia (SADS-IV) and the Structured Clinical Interview for DSM-5 Disorders (SCID-5), followed by clinical consensus with a psychiatrist blind to offspring psychopathology.

\section{Offspring assessment}

\section{Cold executive function: spatial working memory}

We measured working memory with the SWM subtest on the Cambridge Neuropsychological Test Battery (CANTAB) (Sahakian \& Owen, 1992). Participants were required to search for a token 'hidden' under one of a varied number of boxes (between 3 and 10 boxes on increasingly difficult trials). Participants were explicitly told that 'once a blue token has been found under a box, there will never be one in there again, so you must not go back to it'. The participant needs to remember which box they have found a token in while searching. SWM measures four aspects of performance: total errors, between search errors, within search errors, and strategy. Between errors are 
Table 1. Demographic and clinical characteristics of youth with and without psychotic symptoms

\begin{tabular}{|c|c|c|c|c|}
\hline & \multirow{2}{*}{$\begin{array}{l}\text { No psychotic symptoms } \\
(n=87) \\
N(\%)\end{array}$} & \multirow{2}{*}{$\begin{array}{l}\text { Psychotic symptoms } \\
(n=69) \\
N(\%)\end{array}$} & \multicolumn{2}{|c|}{$\begin{array}{l}\text { Comparison of youth } \\
\text { with and without } \\
\text { psychotic symptoms }\end{array}$} \\
\hline & & & $\chi^{2}$ & $p$ value \\
\hline Sex & & & 0.591 & 0.442 \\
\hline Male & $47(54.02)$ & $33(47.83)$ & & \\
\hline Female & $40(45.98)$ & $36(52.17)$ & & \\
\hline Parent primary diagnosis & & 5.391 & 0.145 & \\
\hline None & $22(25.29)$ & $8(11.59)$ & & \\
\hline Depression & $34(39.08)$ & $31(44.93)$ & & \\
\hline Bipolar disorder & $28(32.18)$ & $27(39.13)$ & & \\
\hline Schizophrenia & $3(3.45)$ & $3(4.35)$ & & \\
\hline Parent psychotic symptoms & $19(21.84)$ & $21(30.43)$ & - & - \\
\hline \multicolumn{5}{|l|}{ Offspring diagnoses } \\
\hline ADHD & $18(20.69)$ & $22(31.88)$ & 2.529 & 0.112 \\
\hline Anxiety disorders & $15(17.24)$ & $31(44.93)$ & 14.186 & 0.001 \\
\hline Depression & $4(4.60)$ & $13(18.84)$ & 8.039 & 0.005 \\
\hline \multicolumn{5}{|l|}{ Offspring psychotic symptoms } \\
\hline KSADS & $0(0)$ & $16(23.19)$ & & \\
\hline Funny Feelings & $0(0)$ & $34(49.28)$ & & \\
\hline SIPS & $0(0)$ & $3(4.35)$ & & \\
\hline \multirow[t]{2}{*}{ SPI-CY } & $0(0)$ & $38(55.07)$ & & \\
\hline & Mean (S.D.) & Mean (S.D.) & $t$ statistic & $p$ value \\
\hline Age & $12.15(3.38)$ & $12.45(4.07)$ & -0.503 & 0.616 \\
\hline SES & $3.08(0.47)$ & $2.25(0.63)$ & 3.870 & 0.001 \\
\hline \multicolumn{5}{|l|}{ General intelligence scores } \\
\hline Vocabulary & $101.59(16.04)$ & $100.15(13.49)$ & 0.596 & 0.552 \\
\hline Similarities & $98.12(15.26)$ & 99.70 (13.97) & -0.663 & 0.228 \\
\hline Block design & $98.72(17.02)$ & $99.86(12.65)$ & -0.459 & 0.647 \\
\hline Matrix reasoning & $100.54(17.19)$ & $100.75(12.60)$ & -0.085 & 0.932 \\
\hline FSIQ & $102.02(13.51)$ & $100.06(11.80)$ & 0.948 & 0.345 \\
\hline
\end{tabular}

FSIQ, full-scale intelligence quotient; ADHD, attention-deficit/hyperactivity disorder; SPI-CY, Schizophrenia Proneness Instrument - Child and Youth version; KSADS, Kiddie schedule for affective disorders and schizophrenia; SIPS, structured interview for prodromal syndromes; SES, socioeconomic status.

Note. $N=156$.

defined as times the participant revisits a box in which a token has been previously found. Within errors are defined as the number of times a participant revisits a box already found to be empty during the same search. Strategy is defined as following a predetermined sequence by beginning with a specific box, and then, once a blue token has been found, to return to that same box to start a new search sequence. Based on an a priori plan, we indexed SWM with 'total errors', which are the number of times a box is selected that is certain not to contain a blue token, i.e. as a sum of between errors and within errors, while errors that can be classified as both within and between are only counted once.

\section{Hot executive function: Cambridge gambling task (CGT)}

We measured decision-making in the context of uncertain rewards and losses with the CGT subtest on the CANTAB (Cambridge Cognition, Cambridge, UK, Sahakian \& Owen, 1992). The CGT involves the participant using a touch screen tablet. At each trial, the participant is presented with a row of 10 red and blue boxes at the top of the screen. The number of boxes of each colour is proportionate to the likelihood that a token is under a box of that color. The participant must guess whether a token is hidden inside a red or blue box and bet an amount of points on the choice. A winning choice is rewarded and a losing choice is deducted based on the number of points risked. For 
example, if the participant places a bet of 75 points and chooses red and the token is inside a red box, the participant will be awarded 75 points. However, if the participant chooses red and the token is inside a blue box, the participant loses 75 points. There are two conditions of the task, each with four trials (ascending and descending bet value). In the ascending condition, bets increase from $5 \%$ to $95 \%$ at $2.5 \mathrm{~s}$ intervals. In the descending condition, bets decrease from $95 \%$ to $5 \%$ at $2.5 \mathrm{~s}$ intervals. The CGT measures six aspects of performance: deliberation time, risk taking, delay aversion, quality of decision-making, and risk adjustment. Deliberation time is the mean time (ms) from the presentation of boxes until a bet is selected. Risk taking measures the mean proportion of points bet on each trial when the more likely outcome is selected. Delay aversion is the difference between the amount of points risked in the descending condition $v$. the ascending condition. Quality of decision-making is calculated as the proportion of trials on which the participant chose to bet on the more likely outcome. Risk adjustment measures the extent to which a participant modulates their risk taking in response to the ratio of red to blue boxes (likelihood of success). In the CGT, there is always potential for losing a large percentage of acquired points in the face of a ratio, which appears to be a winning choice, and participants learn this on the practice trial. Therefore, even after learning the rules of the game, the participants need to modulate their behavior in the face of potential gain and loss. For example, they must consider whether or not they want to risk betting $95 \%$ of their points on a ratio with a high likelihood of success $(9: 1 ; 8: 2)$, or whether a more balanced bet is wise considering potential loss. Thus, participants must maintain effective modulation of their decision-making behavior while being aware of potential reward and punishment. The ability to modulate behavior in the face of high and low potential for success is a task with an important emotion component (Bechara, 2004). Based on an a priori analysis plan and consistent with a prior study (Murphy et al. 2001), we have constructed a decision-making score as standardized average of the two measures that specifically index hot decisionmaking: the quality of decision-making and risk adjustment.

\section{Wechsler Abbreviated Scale of Intelligence (WASI)}

We assessed general cognitive ability with the WASI Second Edition (WASI-II; Wechsler, 1999). The WASI-II is an individually administered assessment of intelligence for individuals aged 6-90 years. The WASI-II was administered by trained research staff and graduate students with neuropsychological training. There are four subtests on the WASI-II: block design, vocabulary, matrix reasoning, and similarities. The WASI-II provides a valid and reliable measure of full-scale intelligence quotient (FSIQ).

\section{Assessment of psychotic symptoms}

We assessed psychotic symptoms experienced in the year prior to the assessment with four instruments as previously reported (MacKenzie et al. 2016): the Kiddie Schedule for Affective Disorders and Schizophrenia, Present and Lifetime version (K-SADS-PL), the funny feelings (FF) interview, structured interview for prodromal syndromes (SIPS) and the Schizophrenia Proneness Instrument - Child and Youth Version (SPI-CY). The assessment was repeated in yearly intervals with a median of two assessments completed per individual (range 1-4 assessments).

Kiddie Schedule for Affective Disorders and Schizophrenia, Present and Lifetime version. Interviewers blind to parent psychopathology assessed youth psychopathology with K-SADS-PL (Kaufman et al. 1997) and established the diagnoses of mental disorders based on DSM-IV criteria. Diagnoses were confirmed in consensus meetings with a child and adolescent psychiatrist who was also blind to information on parent psychopathology. We used the K-SADS interview psychosis module and appendix to assess psychotic symptoms, which were also consensus rated by the child and adolescent psychiatrist blind to parent psychopathology. As in our previous publication (MacKenzie et al. 2016), we only included psychotic symptoms classified as 'definite'(K-SADS rating =3).

Funny Feelings (Poulton et al. 2000; Arseneault et al. 2011). We assessed psychotic symptoms with the 'FF' interview where the answers to seven standard questions are corroborated with probes and independent clinical curation (Arseneault et al. 2011). We recorded frequency, distress, impairment, and appraisal (internal/ external, significant/not-significant) for each recent symptom. We submitted the verbatim transcript of each reported experience for independent clinical curation (blind to parent psychopathology) to establish the psychotic nature of the experiences, rated as none, probable, or definite. In analyses, we only utilized psychotic symptoms rated as definite by consensus between two independent raters (MacKenzie et al. 2016).

Structured Interview for Prodromal Syndromes (Miller et al. 1995). In participants aged 11 and above, we also assessed psychotic symptoms with the SIPS, which allows the derivation of attenuated psychotic 
illness and definition of 'at-risk mental state' for psychosis (Miller et al. 1995). In analyses, we only considered SIPS ratings of 3 and above that meet the threshold for at-risk mental state.

Schizophrenia Proneness Instrument - Child and Youth Version (Fux et al. 2013). We interviewed participants aged 8-21 with the SPI-CY to assess basic symptoms. Basic symptoms are subjectively perceived deficits and abnormalities in multiple domains (perception, physical sensation, language, feelings) and often represent early manifestations of psychosis. Basic symptoms have been shown to strongly and specifically predict the development of schizophrenia (Fux et al. 2013). In analyses, we only considered basic symptoms fulfilling criteria for the high-risk profiles of cognitive disturbances (COGDIS) or cognitive-perceptive basic symptoms (COPER) that were shown to predict psychosis with high specificity (Schimmelmann et al. 2013).

\section{Socioeconomic status (SES)}

We indexed SES with a composite variable created as a sum of five dichotomous indicators of maternal and paternal levels of education (higher than high school), whether the family owns or rents their primary residence, household annual income (above $\$ 40000$ / year), as well as the ratio of bedrooms in the home to the number of persons living in the households (one or higher). Each component was converted to a dichotomous variable based on a median cut-off $(0=$ does not meet criteria, $1=$ meets criteria) before summing, resulting in an SES score that ranges from 0 to 5 .

\section{Data analysis}

The primary dependent variable was the presence of one or more definite psychotic symptom, a composite binary variable, defined as at least one of the following: (1) definite clinically significant hallucinations or delusions established with the K-SADS interview; (2) unusual experiences reported on FF and confirmed as 'definite' psychotic symptoms through independent clinical curation; (3) positive symptoms on SIPS reaching threshold for at-risk mental state; (4) high-risk basic symptom profiles COGDIS or COPER on the SPI-CY. The primary independent variable of cold executive function was the total error score from SWM. The primary independent variable of hot executive function was the decision-making score from CGT. We quantified pairwise correlations between CGT measures, SWM measures, and FSIQ as the Pearson productmoment correlation coefficient $(r)$. The CGT and SWM variables were $z$-score standardized to a mean of 0 and standard deviation of 1 and coded, so that higher scores indicate worse performance. We tested the relationships between executive functions and psychotic symptoms using mixed-effect logistic regression applied in the generalized linear latent and mixed model (GLLAMM; (Rabe-Hesketh and Skrondal, 2016), which allows inclusion of repeated assessments from the same individual and accounts for nonindependence of observations from related individuals with nested random effects of individual and family. All analyses controlled for participant's age and sex as fixed-effect covariates. In addition, we conducted sensitivity analyses using lifetime diagnosis of attention-deficit hyperactivity disorder (ADHD), any anxiety disorder, major depressive disorder, FSIQ, SES, and family history of psychotic symptoms as additional covariates. Effect sizes are reported as odds ratios (ORs) with 95\% confidence intervals (95\% CIs). We report associations with a $p$ value smaller than 0.05 as statistically significant.

\section{Results \\ Sample}

Of the 156 participants aged 7-24, 69 (44.23\%) met criteria for at least one definite psychotic symptom on one or more assessments. Table 1 presents demographic and descriptive characteristics of the participants with and without psychotic symptoms. There was no significant difference in FSIQ between participants with and without psychotic symptoms $(t=0.948, p=$ 0.345; Table 1).

\section{Relationships between measures of cognitive ability}

The measures of hot and cold executive functions were only moderately correlated with one another $(r \leqslant 0.40)$ and weakly correlated with FSIQ $(r<0.30$, Table 2).

\section{Cold executive function: SWM and psychotic symptoms}

SWM total errors were not significantly associated with increased risk of psychotic symptoms (OR 1.38, $95 \%$ CI $0.87-2.18, p=0.168$; Fig. 1). None of the component measures from SWM was significantly associated with psychotic symptoms (Table 3).

\section{Hot executive function: decision-making and psychotic symptoms}

Worse hot decision-making score was associated with increased risk of psychotic symptoms (OR 2.37, 95\% CI 1.25-4.50, $p=0.008$; Fig. 1). Further exploratory analyses suggested that psychotic symptoms were significantly associated with worse performance on both 
Table 2. Correlations between Cambridge gambling task performance, spatial working memory performance, and general cognitive ability

\begin{tabular}{|c|c|c|c|c|c|c|c|c|c|c|c|}
\hline & 1 & 2 & 3 & 4 & 5 & 6 & 7 & 8 & 9 & 10 & 11 \\
\hline \multicolumn{12}{|c|}{ Cambridge Gambling Task } \\
\hline 1. Deliberation time & 1 & & & & & & & & & & \\
\hline 2. Risk taking & 0.03 & 1 & & & & & & & & & \\
\hline 3. Delay aversion & -0.25 & -0.03 & 1 & & & & & & & & \\
\hline 4. Quality & -0.34 & -0.1 & -0.04 & 1 & & & & & & & \\
\hline 5. Risk adjustment & -0.31 & -0.32 & -0.11 & 0.28 & 1 & & & & & & \\
\hline 6. DM score & 0.43 & 0.26 & 0.08 & -0.82 & -0.07 & 1 & & & & & \\
\hline 7. Total errors & 0.19 & 0.23 & 0.13 & -0.29 & -0.33 & 0.38 & 1 & & & & \\
\hline \multicolumn{12}{|c|}{ Spatial Working Memory } \\
\hline 8. Between errors & 0.19 & 0.23 & 0.13 & -0.3 & -0.33 & 0.39 & 0.99 & 1 & & & \\
\hline 9. Within errors & 0.07 & -0.01 & 0.17 & 0.03 & -0.1 & 0.03 & 0.45 & 0.4 & 1 & & \\
\hline 10. Strategy & 0.20 & 0.15 & 0.17 & -0.32 & -0.31 & 0.40 & 0.64 & 0.65 & 0.22 & 1 & \\
\hline 11. FSIQ & -0.06 & 0.04 & -0.04 & 0.14 & 0.16 & -0.15 & -0.27 & -0.28 & -0.11 & -0.30 & 1 \\
\hline
\end{tabular}

Quality, quality of decision-making; DM score, decision-making score; Total errors, spatial working memory total errors; Between errors, spatial working memory between errors; Within errors, spatial working memory within errors; Strategy, spatial working memory strategy; FSIQ, full-scale intelligence quotient.

Note. The numbers in the top row correspond to the number of variables in the first column. The numbers below the diagonal in the body of the table are Pearson product-moment correlation coefficients reflecting pairwise correlations between variables in the entire sample $(N=156)$

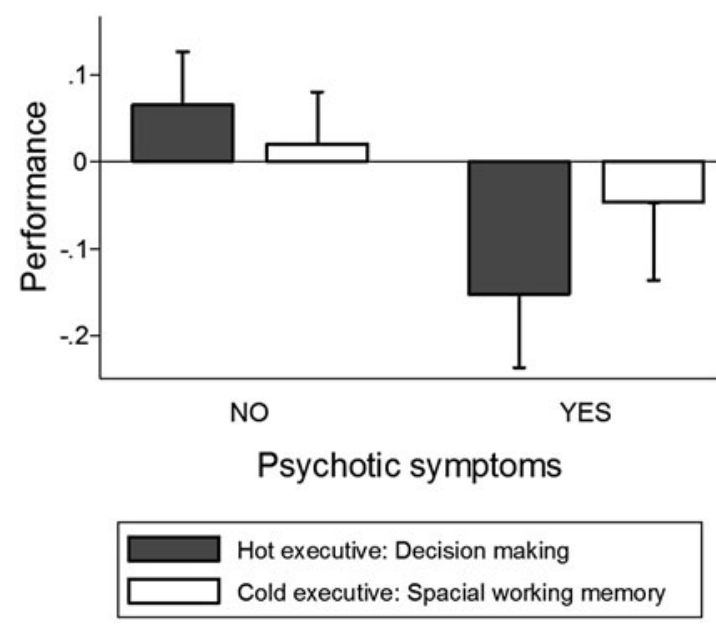

Fig. 1. Performance on hot and cold executive function tests by individuals with and without psychotic symptoms. Note. Measures of performance are standardized so that 0 corresponds to the sample mean and standard deviation is 1.00. Error bars reflect one standard error.

components of hot decision-making, the quality of decision-making, and risk adjustment, but not with other measures from the CGT (Table 3). In sensitivity analyses, the association between poor hot decisionmaking and psychotic symptoms remained significant in models controlling for FSIQ, SES, ADHD, any anxiety disorder, and major depressive disorder in youth and psychotic symptoms in parents. The association between poor hot decision-making and psychotic symptoms also remained significant in a model that controlled for cold executive function (SWM total errors) as a covariate (OR 2.20, 95\% CI 1.14-4.24, $p=0.019$ ).

\section{Discussion}

In a sample of youth enriched for familial risk of psychopathology, we found a robust association between hot executive function and psychotic symptoms. The association was specific to the type of executive functions that required adjusting decisions according to the probability of uncertain rewards and losses. Individuals who were less able to select the more likely outcome and adjust their bet to the probability of reward were more likely to experience psychotic symptoms. The association remained evident after general cognitive ability and cold executive function were accounted for. We found no significant association between psychotic symptoms and cold executive function. These findings suggest that poor hot executive functioning is specifically associated with the propensity for psychotic symptoms.

Our findings have implications for the understanding of the cognitive-emotional mechanisms that may underlie the propensity to psychotic symptoms. Earlier work suggested that emotional appraisal and emotional salience of unusual experiences are key mechanisms leading to the development and maintenance of psychotic symptoms (Underwood et al. 2015; 
Table 3. Performance on the Cambridge gambling task and spatial working memory task in youth with and without psychotic symptoms

\begin{tabular}{|c|c|c|c|c|c|c|c|}
\hline & \multicolumn{2}{|c|}{$\begin{array}{l}\text { Youth without } \\
\text { psychotic symptoms }\end{array}$} & \multicolumn{2}{|c|}{$\begin{array}{l}\text { Youth with psychotic } \\
\text { symptoms }\end{array}$} & \multicolumn{3}{|c|}{$\begin{array}{l}\text { Effects of performance on the risk of } \\
\text { experiencing psychotic symptoms }\end{array}$} \\
\hline & Mean & S.D. & Mean & S.D. & Odds ratio & $95 \% \mathrm{CI}$ & $p$ value \\
\hline \multicolumn{8}{|l|}{ Cambridge Gabling Task } \\
\hline Deliberation time & 2825.1 & 987.6 & 2945.5 & 1152.0 & 0.843 & $0.533-1.335$ & 0.469 \\
\hline Risk taking & 0.567 & 0.171 & 0.587 & 0.168 & 0.918 & $0.585-1.440$ & 0.712 \\
\hline Delay aversion & 0.276 & 0.223 & 0.264 & 0.231 & 1.176 & $0.785-1.760$ & 0.429 \\
\hline Quality of decision-making & 0.846 & 0.146 & 0.805 & 0.167 & 1.665 & $1.035-2.676$ & 0.035 \\
\hline Risk adjustment & 0.863 & 1.122 & 0.762 & 0.894 & 1.732 & $1.020-2.938$ & 0.041 \\
\hline DM score & -0.057 & 0.804 & 0.126 & 0.811 & 2.370 & $1.247-4.504$ & 0.008 \\
\hline \multicolumn{8}{|l|}{ Spatial Working Memory } \\
\hline Total errors & 45.544 & 19.232 & 46.906 & 19.667 & 1.379 & $0.872-2.178$ & 0.168 \\
\hline Between errors & 44.714 & 18.871 & 46.372 & 19.307 & 1.478 & $0.933-2.341$ & 0.095 \\
\hline Within errors & 2.642 & 3.512 & 2.232 & 2.750 & 0.844 & $0.542-1.315$ & 0.455 \\
\hline Strategy & 28.803 & 4.158 & 28.674 & 5.153 & 1.048 & $0.952-1.154$ & 0.331 \\
\hline
\end{tabular}

95\% CI, 95\% confidence interval; DM score, decision-making score.

Note. $N=156$. Regression results are from a GLLAMM model adjusted for age, sex, family history of psychotic symptoms, and familial clustering.

Reininghaus et al. 2016). The present finding extends our understanding of the underlying mechanism to the ability to make rational decisions in the context of rewards and punishments that are unrelated to unusual experiences. This may point to more general cognitive mechanisms that may be linked to neurodevelopment and genetic factors. The specificity of the association to hot as opposed to cold cognition suggests the involvement of emotional-cognitive networks, including the subgenual anterior cingulate cortex, nucleus accumbens, amygdala, and hippocampus (Roiser \& Sahakian, 2013).

Our findings are consistent with a previous study, which found impaired hot decision-making among individuals in their first episode of schizophrenia (Hutton et al. 2002). Our results extend those findings to suggest that the deficit in hot decision-making predates the development of psychotic illness. Deficits that are already present early in development and associated with psychotic symptoms outside the context of SMI are more likely to be related to the causal mechanisms and more useful in the early identification of individuals at risk. In contrast with previous findings in individuals with schizophrenia (Hutton et al. 2002), we have not seen any difference in the latency of decisionmaking. Notably, Hutton et al. found that the latencies were longer in established illness than in first episodes in patients. This pattern of findings suggest that while the hot decision-making may be an early marker of vulnerability, the slowing of performance speed may be a secondary downstream effect of the illness.
Our finding of no significant association between cold executive functioning and psychotic symptoms should be interpreted in the context of published literature and with regard to the limited statistical power of our study. In a large general population sample, Niarchou et al. (2013) reported that decreased processing speed and attention at age 8-11 were associated with psychotic symptoms at age 12 , albeit with a small effect size. This is consistent with a nonsignificant trend in our data for increased odds of experiencing psychotic symptoms in youth who make more errors on the SWM task. However, our study did not have sufficient statistical power to detect small effect sizes. In the context of published literature, the most likely interpretation is that the cold executive functioning may be associated with the propensity to experience psychotic symptoms, but this association is much weaker than the relationship with hot executive function.

In patients with psychotic disorder, neurocognitive deficits have greater impact on vocational and social outcomes than negative and positive symptoms of psychosis (Green, 1996). In spite of the established relevance of hot executive function for real-world functioning ( $\mathrm{Li}$ et al. 2014), research investigating cognitive deficits in those at risk for psychotic disorders primarily relies on traditional neuropsychological tests, often omitting hot executive functioning. Our findings add to the existing evidence by suggesting that hot executive function may index the risk for developing psychotic symptoms. Future investigations 
should include measures of hot emotional functions to complement existing cognitive batteries in the identification of individuals at risk for developing SMI and in need for pre-emptive early interventions. Evidence indicates that it is possible to remediate executive functions through early interventions (Diamond \& Lee, 2011). It remains to be established whether hot executive function may be a modifiable risk factor amenable to change through training or cognitive remediation.

Our findings should be interpreted in the context of several limitations. First, even with a longitudinal study design, it was not possible to determine the direction of cause and effect. This is due to the fact that psychotic symptoms commonly occur in childhood and tend to be persistent. None of the youth in our sample had a diagnosis of psychosis at the time of completing the cognitive tasks. However, a large proportion of the psychotic symptom group experienced psychotic symptoms both before and after completing the cognitive task. Therefore, it was not possible to unequivocally determine whether deficits in hot decision-making are associated with increased propensity to experience psychotic symptoms or exclude the possibility that psychotic symptoms lead to deficits in hot decision-making. In the absence of impairing illness, we believe that it is more likely that poor hot executive cognitive ability makes an individual more prone to psychotic symptoms than psychotic symptoms causing impairment in cognition. However, a definite answer to this question may require mapping of hot cognitive function development across childhood. Second, the present sample primarily comprised youth at familial high risk for SMI, among whom psychotic symptoms are more prevalent than in the general population (Zammit et al. 2013, 2014). Therefore, it remains to be established if the relationship between poor hot executive functioning and psychotic symptoms generalizes to individuals without family history of SMI. Third, although our study was sufficiently powered to detect the moderate effect sizes found in the hot executive function domain, it was underpowered to detect small effect sizes that might reflect a weaker relationship between psychotic symptoms and cold executive function. This was especially noticeable when comparing our cold executive functioning effect size (OR 1.38, 95\% CI 0.87-2.18) with previous reports of a significantly increased propensity to experience psychotic symptoms in youth with decreased cold executive functioning performance (processing speed at age 8: OR 1.24, 95\% CI 1.12-1.36 and attention at age 11: OR 1.14, 95\% CI 1.04-1.25) (Niarchou et al. 2013). In a study of psychotic symptoms and hot and cold executive functions, a large and complete population-based cohort will be needed to establish the degree of specificity in the association of psychotic symptoms with hot $v$. cold executive functioning.

In conclusion, the present findings indicate that impaired hot decision-making is associated with increased propensity to experience psychotic symptoms. Since psychotic symptoms are strongly associated with later development of SMI (Poulton et al. 2000; Welham et al. 2009; Kelleher et al. 2010), impaired hot decision-making may be a neurocognitive marker of SMI risk. Further research in additional domains of hot executive functions is needed to investigate potential implications for the development of SMI and examining hot decision-making as a potential target for pre-emptive early interventions.

\section{Acknowledgements}

The authors would like to thank the families who participated in FORBOW. The authors would also like to acknowledge the contributions of the FORBOW research team, and its steering and advisory committee (see http://www.forbow.org).This work has been supported by the Canadian Institutes of Health Research (Grant reference numbers 124976, 142738, and 148394), the Canada Foundation for Innovation, the Nova Scotia Health Research Foundation and the Dalhousie Medical Research Foundation. Dr H. L. Fisher is supported by an MQ Fellows Award (MQ14F40). Dr R. Uher is supported by the Canada Research Chairs Program.

\section{Declaration of Interest}

None.

\section{Ethical Standards}

The authors assert that all procedures contributing to this work comply with the ethical standards of the relevant national and institutional committees on human experimentation and with the Helsinki Declaration of 1975, as revised in 2008.

\section{References}

Arseneault L, Cannon M, Fisher HL, Polanczyk G, Moffit TE, Caspi A (2011). Childhood trauma and children's emerging psychotic symptoms: a genetically sensitive longitudinal cohort study. American Journal of Psychiatry 168, 65-72.

Bechara A (2004). The role of emotion in decision-making: evidence from neurological patients with orbitofrontal damage. Brain and Cognition 55, 30-40.

Bechara A, Damasio H, Damasio AR (2000). Emotion, decision making and the orbitofrontal cortex. Cerebral Cortex 10, 295-307. 
Bechara A, Damasio H, Damasio AR, Lee GP (1999). Different contributions of the human amygdala and ventromedial prefrontal cortex to decision-making. Journal of Neuroscience 19, 5473-5481.

Blanchard MM, Jacobson S, Clarke MC, Connor D, Kelleher I, Garavan H, Harley M, Cannon M (2010). Language, motor and speed of processing deficits in adolescents with subclinical psychotic symptoms. Schizophrenia Research 123, 71-76.

Bora E, Harrison BJ, Yücel M, Pantelis C (2013). Cognitive impairment in euthymic major depressive disorder: a meta-analysis. Psychological Medicine 43, 2017-2026.

Bora E, Pantelis C (2015). Meta-analysis of cognitive impairment in first-episode bipolar disorder: comparison with first-episode schizophrenia and healthy controls. Schizophrenia Bulletin 41, 1095-1104.

Bora E, Yucel M, Pantelis C (2009). Cognitive endophenotypes of bipolar disorder: a meta-analysis of neuropsychological deficits in euthymic patients and their first-degree relatives. Journal of Affective Disorders 113, $1-20$.

Chan RCK, Shum D, Toulopoulou T, Chen EYH (2008). Assessment of executive functions: review of instruments and identification of critical issues. Archives of Clinical Neuropsychology 23, 201-216.

Cullen AE, Dickson H, West SA, Morris RG, Mould GL, Hodgins S, Murray RM, Laurens KR (2010).

Neurocognitive performance in children aged 9-12 years who present putative antecedents of schizophrenia. Schizophrenia Research 121, 15-23.

Diamond A, Lee K (2011). Interventions shown to aid executive function development in children 4 to 12 years old. Science 333, 959-964.

Dickson H, Cullen AE, Reichenberg A, Hodgins S, Campbell DD, Morris RG, Laurens KR (2014). Cognitive impairment among children at-risk for schizophrenia. Journal of Psychiatric Research 50, 92-99.

Fisher HL, Caspi A, Poulton R, Meier MH, Houts R, Harrington H, Arseneault L, Moffitt TE (2013). Specificity of childhood psychotic symptoms for predicting schizophrenia by 38 years of age: a birth cohort study. Psychological Medicine 43, 2077-2086.

Fux L, Walger P, Schimmelmann BG, Schultze-Lutter F (2013). The schizophrenia proneness instrument, child and youth version (SPI-CY): practicability and discriminative validity. Schizophrenia Research 146, 69-78.

Grafman J, Litvan I (1999). Importance of deficits in executive functions. Lancet 354, 1921-1923.

Green MF (1996). What are the functional consequences of neurocognitive deficits in schizophrenia? [see comments]. American Journal of Psychiatry 153, 321-330.

Hutton SB, Murphy FC, Joyce EM, Rogers RD, Cuthbert I, Barnes TRE, McKenna PJ, Sahakian BJ, Robbins TW (2002). Decision making deficits in patients with first-episode and chronic schizophrenia. Schizophrenia Research 55, 249-257.

Jameson KG, Nasrallah HA, Northern TG, Welge JA (2011). Executive function impairment in first-degree relatives of persons with schizophrenia: a meta-analysis of controlled studies. Asian Journal of Psychiatry 4, 96-99.

Kaufman J, Birmaher B, Brent D, Rao U, Flynn C, Moreci P, Williamson D, Ryan ND (1997). Schedule for Affective Disorders and Schizophrenia for School-Age Children-Present and Lifetime Version (K-SADS-PL): initial reliability and validity data. Journal of the American Academy of Child and Adolescent Psychiatry 36, 980-988.

Kelleher I, Connor D, Clarke MC, Devlin N, Harley M, Cannon M (2012a). Prevalence of psychotic symptoms in childhood and adolescence: a systematic review and meta-analysis of population-based studies. Psychological Medicine 42, 1857-1863.

Kelleher I, Jenner JA, Cannon M (2010). Psychotic symptoms in the general population - An evolutionary perspective. British Journal of Psychiatry 197, 167-169.

Kelleher I, Murtagh A, Clarke MC, Murphy J, Rawdon C, Cannon M (2012b). Neurocognitive performance of a community-based sample of young people at putative ultra high risk for psychosis: support for the processing speed hypothesis. Cognitive Neuropsychiatry 18, 1-17.

Li Y, Cao F, Shao D, Xue J (2014). Ecological assessment of executive functions in adolescents genetically at high risk for schizophrenia. Comprehensive Psychiatry 55, 1350-1357.

MacKenzie LE, Abidi S, Fisher HL, Propper L, Bagnell A, Morash-Conway J, Glover JM, Cumby J, Hajek T, Schultze-Lutter F, Pajer K, Alda M, Uher R (2016). Stimulant medication and psychotic symptoms in offspring of parents with mental illness. Pediatrics 137, e20152486.

Miller TJ, Mcglashan TH, Rosen JL, Cadenhead K, Ventura J, Mcfarlane W, Perkins DO, Pearlson GD, Woods SW (1995). Prodromal assessment with the structured interview for prodromal syndromes and the scale of prodromal symptoms: predictive validity, interrater reliability, and training to reliability. Schizophrenia Bulletin 29, 703-716.

Murphy FC, Rubinsztein JS, Michael A, Rogers RD, Robbins TW, Paykel ES, Sahakian BJ (2001).

Decision-making cognition in mania and depression. Psychological Medicine 31, 679-693.

Niarchou M, Zammit S, Walters J, Lewis G, Owen MJ, Owen MJ, van den Bree MB (2013). Defective processing speed and nonclinical psychotic experiences in children: longitudinal analyses in a large birth cohort. American Journal of Psychiatry 170, 550-557.

Polanczyk G, Moffitt TE, Arseneault L, Cannon M, Ambler A, Richard SEK, Houts R, Odgers CL, Caspi A (2010). Etiological and clinical features of childhood psychotic symptoms: results from a birth cohort. Archives of General Psychiatry 67, 328-338.

Poulton R, Caspi A, Moffitt TE, Cannon M, Murray R, Harrington H (2000). Children's self-reported psychotic symptoms and adult schizophreniform disorder: a 15-year longitudinal study. Archives of General Psychiatry 57, 1053-1058.

Rabe-Hesketh S, Skrondal A (2016). Generalized linear latent and mixed modeling. In Handbook of Item Response Theory: Models, Statistical Tools, and Applications (Vol. I) (ed. W.J. van der Linden), pp. 503-526. CRC Press: Boca Raton. 
Reininghaus U, Kempton MJ, Valmaggia L, Craig TKJ, Garety P, Onyejiaka A, Gayer-Anderson C, So SH, Hubbard K, Beards S, Dazzan P, Pariante C, Mondelli V, Fisher HL, Mills JG, Viechtbauer W, McGuire P, van Os J, Murray RM, Wykes T, Myin-Germeys I, Morgan C (2016). Stress sensitivity, aberrant salience, and threat anticipation in early psychosis: an experience sampling study. Schizophrenia Bulletin 42, 712-722.

Roiser JP, Sahakian BJ (2013). Hot and cold cognition in depression. CNS Spectrums 18, 139-149.

Rushworth MFS, Owen AM (1998). The functional organization of the lateral frontal cortex: conjecture of conjuncture in the electrophysiology literature? Trends in Cognitive Sciences 2, 46-53.

Sahakian BJ, Owen AM (1992). Computerized assessment in neuropsychiatry using CANTAB: discussion paper. Journal of the Royal Society of Medicine 85, 399-402.

Schimmelmann B, Walger P, Schultze-Lutter F (2013). The significance of at-risk symptoms for psychosis in children and adolescents. Canadian Journal of Psychiatry. Revue Canadienne de Psychiatrie 58, 32-40.

Snyder HR (2013). Major depressive disorder is associated with broad impairments on neuropsychological measures of executive function: a meta-analysis and review. Psychological Bulletin 139, 81-132.

Uher R, Cumby J, MacKenzie LE, Morash-Conway J, Glover JM, Aylott A, Propper L, Abidi S, Bagnell A, Pavlova B, Hajek T, Lovas D, Pajer K, Gardner W, Levy A, Alda M
(2014). A familial risk enriched cohort as a platform for testing early interventions to prevent severe mental illness. BMC Psychiatry 14, 344.

Underwood R, Peters E, Kumari V (2015). Psychobiology of threat appraisal in the context of psychotic experiences: a selective review. European Psychiatry 30, 817-829.

Wechsler D (1999). Wechsler Abbreviated Scale of Intelligence. Psychological Corporation: San Antonio.

Welham J, Scott J, Williams G, Najman J, Bor W, O'Callaghan M, McGrath J (2009). Emotional and behavioural antecedents of young adults who screen positive for non-affective psychosis: a 21-year birth cohort study. Psychological Medicine 39, 625-634.

Westerhausen R, Kompus K, Hugdahl K (2011). Impaired cognitive inhibition in schizophrenia: a meta-analysis of the Stroop interference effect. Schizophrenia Research 133, 172-181.

Zammit S, Hamshere M, Dwyer S, Georgiva L, Timpson N, Moskvina V, Richards A, Evans DM, Lewis G, Jones P, Owen MJ, O'Donovan MC (2014). A population-based study of genetic variation and psychotic experiences in adolescents. Schizophrenia Bulletin 40, 1254-1262.

Zammit S, Kounali D, Cannon M, David AS, Gunnell D, Heron J, Jones PB, Lewis S, Sullivan S, Wolke D, Lewis G (2013). Psychotic experiences and psychotic disorders at age 18 in relation to psychotic experiences at age 12 in a longitudinal population-based cohort study. American Journal of Psychiatry 170, 742-750. 